\title{
A NOTE ON PARTIALLY ORDERED FUZZY METRIC SPACE VIA COUPLED COINCIDENCE POINTS
}

\author{
Arihant Jain ${ }^{1}$ and Vaijayanti Supekar ${ }^{2}$ \\ ${ }^{1}$ Department of Applied Mathematics, Shri Guru Sandipani Institute of Technology and Science, \\ Ujjain (M.P.) 456550 India \\ ${ }^{2}$ Research Scholar, Mewar University, Chittorgarh (Rajasthan), India
}

ABSTRACT. In this paper, we prove a coupled coincidence point theorem in partially ordered fuzzy metric space using $\phi$-contractive condition.

2000 MATHEMATICS SUBJECT CLASSIFICATION. 54H25, 47H10.

KEYWORDS AND PHRASES. Coupled coincidence point; partially ordered set; fuzzy metric space; mixed monotone mapping.

\section{Council for Innovative Research}

Peer Review Research Publishing System

Journal: J OURNAL OF ADVANCES IN MATHEMATICS

Vol.11, No. 5

www.cirjam.com, editorjam@gmail.com 


\section{INTRODUCTION}

After Zadeh [8] introduced the concept of fuzzy sets in 1965, many authors have extensively developed the theory of fuzzy sets and its applications. Specially to mention, fuzzy metric spaces were introduced by Deng [2], Erceg [3], Kaleva and Seikkala [5], Kramosil and Michalek [6]. In this paper we use the concept of fuzzy metric space introduced by Kramosil and Michalek [6] and modified by George and Veeramani [4] to obtain Hausdorff topology for this kind of fuzzy metric space.

Recently, Bhaskar and Lakshmikantham [1] introduced the concept of mixed monotone mapping and coupled fixed point in complete metric space. After that, Lakshmikantham and Ćirić [7] gave a new concept of commutative mapping with mixed monotone property in complete metric space which was the extended and improved version of result of Bhaskar and Lakshmikantham [1]. In the present paper, inspired by the result of Lakshmikantham and Ćirić [7], we prove coupled common fixed point theorems in a partially ordered fuzzy metric space.

\section{PRELIMINARIES}

In this section we recall some definitions and known results in fuzzy metric space.

Definition 2.1. [4] A binary operation $*:[0,1] \times[0,1] \rightarrow[0,1]$ is called a t-norm if $([0,1], *)$ is an abelian topological monoid with unit 1 such that $\mathrm{a} * \mathrm{~b} \leq \mathrm{c} * \mathrm{~d}$ whenever $\mathrm{a} \leq \mathrm{c}$ and $\mathrm{b} \leq \mathrm{d}$ for $\mathrm{a}, \mathrm{b}, \mathrm{c}, \mathrm{d} \in[0,1]$.

$$
\text { Examples of } \mathrm{t} \text {-norms are } \mathrm{a} * \mathrm{~b}=\mathrm{ab} \quad \text { and } \mathrm{a} * \mathrm{~b}=\min \{\mathrm{a}, \mathrm{b}\} \text {. }
$$

Definition 2.2. [4] The 3-tuple $(X, M, *)$ is said to be a Fuzzy metric space if Xis an arbitrary set, * is a continuous t-norm and $\mathrm{M}$ is a Fuzzy set in $\mathrm{X}^{2} \times[0, \infty)$ satisfying the following conditions :

for all $\mathrm{x}, \mathrm{y}, \mathrm{z} \in \mathrm{X}$ and $\mathrm{s}, \mathrm{t}>0$.

(FM-1) $\mathrm{M}(\mathrm{x}, \mathrm{y}, 0)=0$,

(FM-2) $\quad M(x, y, t)=1$ for all $t>0$ if and only if $x=y$,

(FM-3) $\quad \mathrm{M}(\mathrm{x}, \mathrm{y}, \mathrm{t})=\mathrm{M}(\mathrm{y}, \mathrm{x}, \mathrm{t})$,

(FM-4) $\quad \mathrm{M}(\mathrm{x}, \mathrm{y}, \mathrm{t}) * \mathrm{M}(\mathrm{y}, \mathrm{z}, \mathrm{s}) \leq \mathrm{M}(\mathrm{x}, \mathrm{z}, \mathrm{t}+\mathrm{s})$,

$(\mathrm{FM}-5) \quad \mathrm{M}(\mathrm{x}, \mathrm{y},):.[0, \propto) \rightarrow[0,1]$ is left continuous,

(FM-6) $\quad \lim _{t \rightarrow \infty} M(x, y, t)=1$.

Note that $M(x, y, t)$ can be considered as the degree of nearness between $x$ and $y$ with respect to $t$. We identify $x=y$ with $M(x, y, t)=1$ for all $t>0$. The following example shows that every metric space induces a Fuzzy metric space.

Example 2.1. [4] Let $(X, d)$ be a metric space. Define $a{ }^{*} b=\min \{a, b\}$ and $M(x, y, t)=\frac{t}{t+d(x, y)}$ for all $x, y \in X$ and all $t>0$. Then $\left(X, M,{ }^{*}\right)$ is a Fuzzy metric space. It is called the Fuzzy metric space induced by $d$.

Definition 2.3. [6] A sequence $\left\{x_{n}\right\}$ in a Fuzzy metric space (X, M, *) is said to be a Cauchy sequence if and only if for each $\varepsilon>0, t>0$, there exists $n_{0} \in N$ such that $M\left(x_{n}, x_{m}, t\right)>1-\varepsilon$ for all $n, m \geq n_{0}$.

The sequence $\left\{\mathrm{x}_{\mathrm{n}}\right\}$ is said to converge to a point $\mathrm{x}$ in $\mathrm{X}$ if and only if for each $\varepsilon>0, t>0$ there exists $\mathrm{n}_{0} \in \mathrm{N}$ such that $M\left(x_{n}, x, t\right)>1-\varepsilon$ for all $n \geq n_{0}$.

A Fuzzy metric space $(\mathrm{X}, \mathrm{M}, *)$ is said to be complete if every Cauchy sequence in it converges to a point in it.

Definition 2.4. An element $(x, y) \in X \times X$ is called a

(i) coupled fixed point of the mapping $f: X \times X \rightarrow X$ if $f(x, y)=x, f(y, x)=y$.

(ii) coupled coincidence point of the mappings $f: X \times X \rightarrow X$ and $g: X \rightarrow X$ if $f(x, y)=g(x), f(y, x)=g(y)$.

Definition 2.5. The mapping $\mathrm{f}: \mathrm{X} \times \mathrm{X} \rightarrow \mathrm{X}$ and $\mathrm{g}: \mathrm{X} \rightarrow \mathrm{X}$ are called commutative if $\mathrm{gf}(\mathrm{x}, \mathrm{y})=\mathrm{f}(\mathrm{gx}, \mathrm{gy})$ for all $\mathrm{x}, \mathrm{y} \in \mathrm{X}$.

Definition 2.6. Let $(X, \leq)$ be a partially ordered set and $G: X \times X \rightarrow X$. The mapping $G$ is said to have the mixed monotone property if $\mathrm{G}$ is monotone, non-decreasing in its first argument and is monotone non-increasing in its second argument, i.e., for any $\mathrm{x}, \mathrm{y} \in \mathrm{X}$,

$$
\mathrm{x}_{1}, \mathrm{x}_{2} \in \mathrm{X}, \mathrm{x}_{1} \leq \mathrm{x}_{2} \text { implies } \mathrm{G}\left(\mathrm{x}_{1}, \mathrm{y}\right) \leq \mathrm{G}\left(\mathrm{x}_{2}, \mathrm{y}\right)
$$

and

$$
\mathrm{y}_{1}, \mathrm{y}_{2} \in \mathrm{Y}, \mathrm{y}_{1} \leq \mathrm{y}_{2} \text { implies } \mathrm{G}\left(\mathrm{x}, \mathrm{y}_{1}\right) \leq \mathrm{G}\left(\mathrm{x}, \mathrm{y}_{2}\right) \text {. }
$$

Definition 2.6. Define $\Phi=\left\{\phi: \mathrm{R}^{+} \rightarrow \mathrm{R}^{+}\right\}$, where $\mathrm{R}^{+}=[0,+\infty)$ and each $\phi \in \Phi$ satisfying the following conditions :

$\left(\phi_{1}\right) \quad \phi$ is strict increasing.

$\left(\phi_{2}\right) \quad \phi$ is upper semi-continuous from the right. 
$\left(\phi_{3}\right)$

$$
\sum_{n=0}^{\infty} \phi^{\mathrm{n}}(\mathrm{t})<+\infty \text { for all } \mathrm{t}>0, \text { where } \phi^{\mathrm{n}+1}(\mathrm{t})=\phi\left(\phi^{\mathrm{n}}(\mathrm{t})\right), \mathrm{n} \in \mathrm{N}
$$

Clearly, if $\phi \in \Phi$ then $\phi(\mathrm{t})<\mathrm{t}$ for all $\mathrm{t}>0$.

Definition 2.7. Let $(X, \leq)$ be a partially ordered set and $\mathrm{G}: \mathrm{X} \times \mathrm{X} \rightarrow \mathrm{X}$ and $\mathrm{g}: \mathrm{X} \rightarrow \mathrm{X}$. We say G has mixed g-monotone property if $\mathrm{G}$ is monotone g-nondecreasing in its first argument and is monotone g-nonincreasing in its second argument, i.e. for any $\mathrm{X}$, $\mathrm{y} \in \mathrm{X}$,

$$
\mathrm{x}_{1}, \mathrm{x}_{2} \in \mathrm{X}, \quad \mathrm{g}\left(\mathrm{x}_{1}\right) \leq \mathrm{g}\left(\mathrm{x}_{2}\right) \text { implies that } \mathrm{G}\left(\mathrm{x}_{1}, \mathrm{y}\right) \leq \mathrm{G}\left(\mathrm{x}_{2}, \mathrm{y}\right)
$$

and $\quad \mathrm{y}_{1}, \mathrm{y}_{2} \in \mathrm{X}, \quad \mathrm{g}\left(\mathrm{y}_{1}\right) \leq \mathrm{g}\left(\mathrm{y}_{2}\right)$ implies that $\mathrm{G}\left(\mathrm{x}, \mathrm{y}_{1}\right) \leq \mathrm{G}\left(\mathrm{x}, \mathrm{y}_{2}\right)$.

Remark 2.1. If we assume $\mathrm{g}=\mathrm{I}$, then definition 2.8 reduces to 2.6 .

Lemma 2.1. [4] Let $(X, M, *)$ be a fuzzy metric space. Then for all $x, y \in X, M(x, y, t)$ is a non-decreasing function. Using FM-6, we can prove the following lemmas :

Lemma 2.2. Let $(\mathrm{X}, \mathrm{M}, *)$ be a fuzzy metric space. If there exists $\phi \in \Phi$ such that for all $\mathrm{x}, \mathrm{y} \in \mathrm{X}$,

$$
\mathrm{M}(\mathrm{x}, \mathrm{y}, \phi(\mathrm{t})) \geq \mathrm{M}(\mathrm{x}, \mathrm{y}, \mathrm{t}) \forall \mathrm{t}>0, \text { then } \mathrm{x}=\mathrm{y} .
$$

Lemma 2.3. Let $(X, M, *)$ be a fuzzy metric space. If we define $G_{\lambda}: X \times X \rightarrow R^{+} \cup\{0\}$ by

$$
\mathrm{G}_{\lambda}(\mathrm{x}, \mathrm{y})=\inf \{\mathrm{t}>0, \mathrm{M}(\mathrm{x}, \mathrm{y}, \mathrm{t})>1-\lambda\}
$$

for all $\lambda \in(0,1]$ and $\mathrm{x}, \mathrm{y} \in \mathrm{X}$, then we have

(i) for each $\lambda \in(0,1]$, there exists $\mu \in(0,1]$ such that $G_{\lambda}\left(x_{1}, x_{n}\right) \leq \sum_{i=1}^{n-1} G_{\mu}\left(x_{i}, x_{i+1}\right)$, for all $x_{1}, \ldots, x_{n} \in X$.

(ii) The sequence $\left\{x_{n}\right\}_{n \in N}$ in $X$ is convergent if and only if $G_{\lambda}\left(x_{n}, x\right) \rightarrow 0$ as $n \rightarrow \infty$ for all $\lambda \in(0,1]$.

Proof. (i) For any $\lambda \in(0,1]$, let $\mu \in(0,1]$ and $\mu<\lambda$, so by triangle inequality and for any $\delta>0$, we have

$$
\begin{gathered}
M\left(x_{1}, x_{n}, \sum_{i=1}^{n-1} G_{\mu}\left(x_{i}, x_{i+1}\right)+(n-1) \delta\right) \\
\quad \geq M\left(x_{1}, x_{2}, G\left(x_{1}, x_{2}\right)+\delta\right) * M\left(x_{2}, x_{3}, G\left(x_{2}, x_{3}\right)+\delta\right) * \ldots * M\left(x_{n-1}, x_{n}, G\left(x_{n-1}, x_{n}\right)+\delta\right) \\
\quad \geq \min \{(1-\mu),(1-\mu), \ldots,(1-\mu)\} \geq(1-\lambda), \text { which implies } \\
G_{\lambda}\left(x_{1}, x_{n}\right) \leq G_{\mu}\left(x_{1}, x_{2}\right)+G_{\mu}\left(x_{2}, x_{3}\right)+G_{\mu}\left(x_{3}, x_{4}\right)+\ldots+G_{\mu}\left(x_{n-1}, x_{n}\right)
\end{gathered}
$$$$
\mathrm{G}_{\lambda}\left(\mathrm{x}_{1}, \mathrm{x}_{\mathrm{n}}\right) \leq \sum_{\mathrm{i}=1}^{\mathrm{n}-1} \mathrm{G}_{\mu}\left(\mathrm{x}_{\mathrm{i}}, \mathrm{x}_{\mathrm{i}+1}\right) \text {. }
$$

(ii) Since $M$ is continuous and $G_{\lambda}(x, y)=\inf \{t>0, M(x, y, t)>1-\lambda\}$

we have

$$
M\left(x_{n}, x, \eta\right)>1-\lambda \text { for all } \eta>0 .
$$

This proves the lemma.

Lemma 2.4. Let $(X, M, *)$ be FM-space and $\left\{y_{n}\right\}$ be a sequence in $X$. If there exists $\phi \in \Phi$ such that

$$
\mathrm{M}\left(\mathrm{y}_{\mathrm{n}}, \mathrm{y}_{\mathrm{n}+1}, \phi(\mathrm{t})\right) \geq \mathrm{M}\left(\mathrm{y}_{\mathrm{n}-1}, \mathrm{y}_{\mathrm{n}}, \mathrm{t}\right) * \mathrm{M}\left(\mathrm{y}_{\mathrm{n}}, \mathrm{y}_{\mathrm{n}+1}, \mathrm{t}\right)
$$

for all $\mathrm{t}>0$ and $\mathrm{n}=1,2, \ldots$, then $\left\{\mathrm{y}_{\mathrm{n}}\right\}$ is a Cauchy sequence in $\mathrm{X}$.

Proof. From (A) and for each $\lambda \in(0,1]$ and $n \in \mathbb{N}$, putting $a_{n}=G_{\lambda}\left(y_{n-1}, y_{n}\right)$, we will prove that

$$
\mathrm{a}_{\mathrm{n}+1} \leq \phi\left(\mathrm{a}_{\mathrm{n}}\right), \forall \mathrm{n} \in \mathbb{N} .
$$

Since $\phi$ is upper semi-continuous from right, for given $\varepsilon>0$ and each $a_{n}$, there exists $v_{n}>a_{n}$ such that $\phi\left(v_{n}\right)<\phi\left(a_{n}\right)+\varepsilon$.

From (A), it follows that

$$
\mathrm{v}_{\mathrm{n}}>\mathrm{a}_{\mathrm{n}}=\mathrm{G}_{\lambda}\left(\mathrm{y}_{\mathrm{n}-1}, \mathrm{y}_{\mathrm{n}}\right) \text { that } \mathrm{M}\left(\mathrm{y}_{\mathrm{n}-1}, \mathrm{y}_{\mathrm{n}}, \mathrm{v}_{\mathrm{n}}\right)>1-\lambda \text { for all } \mathrm{n} \in \mathbb{N} .
$$

Thus, by (B), (C) and lemma 2.1, we get

$$
\begin{aligned}
\mathrm{M}\left(\mathrm{y}_{\mathrm{n}}, \mathrm{y}_{\mathrm{n}+1}, \phi\left(\max \left\{\mathrm{v}_{\mathrm{n}}, \mathrm{v}_{\mathrm{n}+1}\right\}\right)\right) \\
\geq \mathrm{M}\left(\mathrm{y}_{\mathrm{n}-1}, \mathrm{y}_{\mathrm{n}}, \max \left\{\mathrm{v}_{\mathrm{n}}, \mathrm{v}_{\mathrm{n}-1}\right\}\right) * \mathrm{M}\left(\mathrm{y}_{\mathrm{n}}, \mathrm{y}_{\mathrm{n}+1}, \max \left\{\mathrm{v}_{\mathrm{n}}, \mathrm{v}_{\mathrm{n}+1}\right\}\right)
\end{aligned}
$$




$$
\begin{aligned}
& \geq M\left(y_{n-1}, y_{n}, v_{n}\right) * M\left(y_{n}, y_{n+1}, v_{n+1}\right) \\
& >1-\lambda .
\end{aligned}
$$

This implies that

$$
\begin{gathered}
\mathrm{G}_{\lambda}\left(\mathrm{y}_{\mathrm{n}}, \mathrm{y}_{\mathrm{n}+1}\right) \leq \phi\left(\max \left\{\mathrm{v}_{\mathrm{n}}, \mathrm{v}_{\mathrm{n}+1}\right\}\right)=\max \left\{\phi\left(\mathrm{v}_{\mathrm{n}}\right), \phi\left(\mathrm{v}_{\mathrm{n}+1}\right)\right\} \\
\leq \max \left\{\phi\left(\mathrm{v}_{\mathrm{n}}\right), \phi\left(\mathrm{v}_{\mathrm{n}+1}\right)\right\}+\varepsilon .
\end{gathered}
$$

By arbitrariness of $\varepsilon$, we have

$$
a_{n+1}=G_{\lambda}\left(y_{n}, y_{n+1}\right) \leq \max \left\{\phi\left(a_{n}\right), \phi\left(a_{n+1}\right)\right\} .
$$

So, we can infer that

$$
\begin{aligned}
& a_{n+1} \leq \phi\left(a_{n}\right) . \text { If not, then by (D), we have } \\
& a_{n+1}<\phi\left(a_{n+1}\right)<a_{n+1} \text {, which is a contradiction. }
\end{aligned}
$$

Hence, (D) implies that

$$
\mathrm{a}_{\mathrm{n}+1} \leq \phi\left(\mathrm{a}_{\mathrm{n}}\right) \text { and }(\mathrm{C}) \text { is proved. }
$$

Now, using (C) again and again, we get

$$
\mathrm{G}_{\lambda}\left(\mathrm{y}_{\mathrm{n}}, \mathrm{y}_{\mathrm{n}+1}\right) \leq \phi\left(\mathrm{G}_{\lambda}\left(\mathrm{y}_{\mathrm{n}-1}, \mathrm{y}_{\mathrm{n}}\right)\right) \leq \ldots \leq \phi^{\mathrm{n}}\left(\mathrm{G}_{\lambda}\left(\mathrm{y}_{0}, \mathrm{y}_{1}\right)\right) \text {, for all } \mathrm{n} \in \mathbb{N} .
$$

By lemma (2.3), for each $\lambda \in(0,1]$, there exists $\mu \in(0, \lambda)$ such that

$$
\mathrm{G}_{\lambda}\left(\mathrm{y}_{\mathrm{n}}, \mathrm{y}_{\mathrm{m}}\right) \leq \sum_{\mathrm{i}=\mathrm{n}}^{\mathrm{m}-1} \mathrm{G}_{\mu}\left(\mathrm{y}_{\mathrm{i}}, \mathrm{y}_{\mathrm{i}+1}\right), \quad \text { for all } \mathrm{m}, \mathrm{n} \in \mathbb{N} \text { with } \mathrm{m}>\mathrm{n} .
$$

Since $\phi \in \Phi$, by condition $\left(\phi_{3}\right)$ we have

$$
\sum_{n=0}^{\infty} \phi^{n}\left(G_{\mu}\left(y_{0}, y_{1}\right)\right)<+\infty
$$

So, for given $\varepsilon>0$, there exists $\mathrm{n}_{0} \in \mathbb{N}$ such that

$$
\sum_{i=n_{0}}^{\infty} \phi^{i}\left(G_{\mu}\left(y_{0}, y_{1}\right)\right)<\varepsilon
$$

Thus, from (E),

$$
\mathrm{G}_{\lambda}\left(\mathrm{y}_{\mathrm{n}}, \mathrm{y}_{\mathrm{m}}\right) \leq \sum_{\mathrm{i}=\mathrm{n}}^{\infty} \phi^{\mathrm{i}}\left(\mathrm{G}_{\mu}\left(\mathrm{y}_{0}, \mathrm{y}_{1}\right)\right)<\varepsilon, \quad \mathrm{n}>\mathrm{n}_{0}
$$

which implies that $\mathrm{M}\left(\mathrm{y}_{\mathrm{n}}, \mathrm{y}_{\mathrm{m}}, \varepsilon\right)>1-\lambda$ for all $\mathrm{m}, \mathrm{n} \in \mathbb{N}$ with $\mathrm{m}>\mathrm{n} \geq \mathrm{n}_{0}$.

Therefore, $\left\{y_{n}\right\}$ is a Cauchy sequence in $X$.

\section{MAIN RESULT.}

Theorem 3.1. Let $(X, M, *)$ be a complete fuzzy metric space and $(X, \leq)$ be a partially ordered set and let $G: X \times X \rightarrow X$ and $\mathrm{g}: \mathrm{X} \rightarrow \mathrm{X}$ be two mappings such that $\mathrm{G}$ has a mixed g-monotone property and there exists $\phi \in \Phi$ such that

$\mathrm{M}(\mathrm{G}(\mathrm{x}, \mathrm{y}), \mathrm{G}(\mathrm{u}, \mathrm{v}), \phi(\mathrm{t})) \geq \mathrm{M}(\mathrm{g}(\mathrm{x}), \mathrm{G}(\mathrm{x}, \mathrm{y}), \mathrm{t}) * \mathrm{M}(\mathrm{g}(\mathrm{u}), \mathrm{G}(\mathrm{u}, \mathrm{v}), \mathrm{t})$

for all $\mathrm{x}, \mathrm{y}, \mathrm{u}, \mathrm{v} \in \mathrm{X}, \mathrm{t}>0$ for which

$$
\mathrm{g}(\mathrm{x}) \geq \mathrm{g}(\mathrm{u}) \text { and } \mathrm{g}(\mathrm{y}) \leq \mathrm{g}(\mathrm{v})
$$

or $\quad \mathrm{g}(\mathrm{x}) \leq \mathrm{g}(\mathrm{u})$ and $\mathrm{g}(\mathrm{y}) \geq \mathrm{g}(\mathrm{v})$.

Suppose $\mathrm{G}(\mathrm{X} \times \mathrm{X}) \subseteq \mathrm{g}(\mathrm{X})$, $\mathrm{g}$ is continuous and commutes with $\mathrm{G}$ and also suppose either

(i) $\mathrm{G}$ is continuous or

(ii) $\mathrm{X}$ has the following properties :

(a) If a non-decreasing sequence $x_{n} \rightarrow x$, then $x_{n} \leq x$, for all $n$ and

(b) If a non-increasing sequence $y_{n} \rightarrow y$, then $y_{n} \geq y$, for all $n$.

If there exist $\mathrm{x}_{0}, \mathrm{y}_{0} \in \mathrm{X}$ such that $\mathrm{g}\left(\mathrm{x}_{0}\right) \leq \mathrm{G}\left(\mathrm{x}_{0}, \mathrm{y}_{0}\right), \mathrm{g}\left(\mathrm{y}_{0}\right) \geq \mathrm{G}\left(\mathrm{y}_{0}, \mathrm{x}_{0}\right)$

then there exist $x, y \in X$ such that $g(x)=G(x, y)$ and $g(y)=G(y, x)$. 
Then $\mathrm{G}$ and $\mathrm{g}$ have a coupled coincidence point in X.

Proof. Let $\mathrm{x}_{0}, \mathrm{y}_{0} \in \mathrm{X}$ be such that $\mathrm{g}\left(\mathrm{x}_{0}\right) \leq \mathrm{G}\left(\mathrm{x}_{0}, \mathrm{y}_{0}\right)$ and $\mathrm{g}\left(\mathrm{y}_{0}\right) \geq \mathrm{G}\left(\mathrm{y}_{0}, \mathrm{x}_{0}\right)$.

Since $\mathrm{G}(\mathrm{X} \times \mathrm{X}) \subseteq \mathrm{g}(\mathrm{X})$, we can choose $\mathrm{x}_{1}, \mathrm{y}_{1} \in \mathrm{X}$ such that

$$
\mathrm{g}\left(\mathrm{x}_{1}\right)=\mathrm{G}\left(\mathrm{x}_{0}, \mathrm{y}_{0}\right) \text { and } \mathrm{g}\left(\mathrm{y}_{1}\right)=\mathrm{G}\left(\mathrm{y}_{0}, \mathrm{x}_{0}\right) \text {. }
$$

Continuing in this way, we can construct sequences $\left\{\mathrm{x}_{\mathrm{n}}\right\}$ and $\left\{\mathrm{y}_{\mathrm{n}}\right\}$ such that

$$
\mathrm{g}\left(\mathrm{x}_{\mathrm{n}+1}\right)=\mathrm{G}\left(\mathrm{x}_{\mathrm{n}}, \mathrm{y}_{\mathrm{n}}\right) \text { and } \mathrm{g}\left(\mathrm{y}_{\mathrm{n}+1}\right)=\mathrm{G}\left(\mathrm{y}_{\mathrm{n}}, \mathrm{x}_{\mathrm{n}}\right) \text {, for all } \mathrm{n} \geq 0 \text {. }
$$

We shall show that

$$
\mathrm{g}\left(\mathrm{x}_{\mathrm{n}}\right) \leq \mathrm{g}\left(\mathrm{x}_{\mathrm{n}+1}\right)
$$

and

$$
\mathrm{g}\left(\mathrm{y}_{\mathrm{n}}\right) \geq \mathrm{g}\left(\mathrm{y}_{\mathrm{n}+1}\right), \text { for all } \mathrm{n} \geq 0 \text {. }
$$

Now, we shall use mathematical induction. Let $\mathrm{n}=0$. Since $\mathrm{g}\left(\mathrm{x}_{0}\right) \leq \mathrm{G}\left(\mathrm{x}_{0}, \mathrm{y}_{0}\right)$ and $\mathrm{g}\left(\mathrm{y}_{0}\right) \geq \mathrm{G}\left(\mathrm{y}_{0}, \mathrm{x}_{0}\right)$ and as $\mathrm{g}\left(\mathrm{x}_{1}\right)=\mathrm{G}\left(\mathrm{x}_{0}, \mathrm{y}_{0}\right)$ and $\mathrm{g}\left(\mathrm{y}_{1}\right)$ $=\mathrm{G}\left(\mathrm{y}_{0}, \mathrm{x}_{0}\right)$, we have

$$
\mathrm{g}\left(\mathrm{x}_{0}\right) \leq \mathrm{g}\left(\mathrm{x}_{1}\right) \text { and } \mathrm{g}\left(\mathrm{y}_{0}\right) \geq \mathrm{g}\left(\mathrm{y}_{1}\right)
$$

Thus, (7) and (8) hold for $\mathrm{n}=0$.

Suppose now that (7) and (8) hold for some fixed point $\mathrm{n} \geq 0$. Then since

$\mathrm{g}\left(\mathrm{x}_{\mathrm{n}}\right) \leq \mathrm{g}\left(\mathrm{x}_{\mathrm{n}+1}\right)$ and $\mathrm{g}\left(\mathrm{y}_{\mathrm{n}}\right) \geq \mathrm{g}\left(\mathrm{y}_{\mathrm{n}+1}\right)$ and as $\mathrm{G}$ has the mixed g-monotone property from (6) and (3)

$$
\left.\begin{array}{l}
g\left(x_{n+1}\right)=G\left(x_{n}, y_{n}\right) \leq G\left(x_{n+1}, y_{n}\right), \\
G\left(y_{n+1}, x_{n}\right) \leq G\left(y_{n}, x_{n}\right)=g\left(y_{n+1}\right)
\end{array}\right\}
$$

and from (6) and (4),

$$
\left.\begin{array}{l}
g\left(x_{n+2}\right)=G\left(x_{n+1}, y_{n+1}\right) \geq G\left(x_{n+1}, y_{n}\right), \\
G\left(y_{n+1}, x_{n}\right) \geq G\left(y_{n+1}, x_{n+1}\right)=g\left(y_{n+2}\right)
\end{array}\right\} .
$$

Now, from (9) and (10), we get

$$
\mathrm{g}\left(\mathrm{x}_{\mathrm{n}+1}\right) \leq \mathrm{g}\left(\mathrm{x}_{\mathrm{n}+2}\right) \text { and } \mathrm{g}\left(\mathrm{y}_{\mathrm{n}+1}\right) \geq \mathrm{g}\left(\mathrm{y}_{\mathrm{n}+2}\right) .
$$

Thus, by mathematical induction, we conclude that (7) and (8) hold for $\mathrm{n} \geq 0$.

Therefore, $\mathrm{g}\left(\mathrm{x}_{0}\right) \leq \mathrm{g}\left(\mathrm{x}_{1}\right) \leq \mathrm{g}\left(\mathrm{x}_{2}\right) \leq \ldots \leq \mathrm{g}\left(\mathrm{x}_{\mathrm{n}}\right) \leq \mathrm{g}\left(\mathrm{x}_{\mathrm{n}+1}\right) \leq \ldots$

and

$$
\mathrm{g}\left(\mathrm{y}_{0}\right) \geq \mathrm{g}\left(\mathrm{y}_{1}\right) \geq \mathrm{g}\left(\mathrm{y}_{2}\right) \geq \ldots \geq \mathrm{g}\left(\mathrm{y}_{\mathrm{n}}\right) \geq \mathrm{g}\left(\mathrm{y}_{\mathrm{n}+1}\right) \geq \ldots
$$

Now, putting $\mathrm{x}=\mathrm{x}_{\mathrm{n}-1}, \mathrm{y}=\mathrm{y}_{\mathrm{n}-1}$ and $\mathrm{u}=\mathrm{x}_{\mathrm{n}}, \mathrm{v}=\mathrm{y}_{\mathrm{n}}$ in equation (5), we get

$M\left(G\left(x_{n-1}, y_{n-1}\right), G\left(x_{n}, y_{n}\right), \phi(t)\right) \geq M\left(g\left(x_{n-1}\right), G\left(x_{n-1}, y_{n-1}\right), t\right) * M\left(g\left(x_{n}\right), G\left(x_{n}, y_{n}\right), t\right)$.

By using (6),

$\mathrm{M}\left(\mathrm{g}\left(\mathrm{x}_{\mathrm{n}}\right), \mathrm{g}\left(\mathrm{x}_{\mathrm{n}+1}\right), \phi(\mathrm{t})\right) \geq \mathrm{M}\left(\mathrm{g}\left(\mathrm{x}_{\mathrm{n}-1}\right), \mathrm{g}\left(\mathrm{x}_{\mathrm{n}}\right), \mathrm{t}\right) * \mathrm{M}\left(\mathrm{g}\left(\mathrm{x}_{\mathrm{n}}\right), \mathrm{g}\left(\mathrm{x}_{\mathrm{n}+1}\right), \mathrm{t}\right)$.

Thus, by lemma 2.4, we conclude $\left\{g\left(x_{n}\right)\right\}$ is a Cauchy sequence in $X$.

Similarly, $\left\{g\left(y_{n}\right)\right\}$ is a Cauchy sequence in $X$.

Since $\mathrm{X}$ is complete, there exist $\mathrm{x}, \mathrm{y} \in \mathrm{X}$ such that

$$
\lim _{n \rightarrow \infty} g\left(x_{n}\right)=x \text { and } \lim _{n \rightarrow \infty} g\left(y_{n}\right)=y .
$$

Since $\mathrm{g}$ is continuous, therefore

$$
\lim _{n \rightarrow \infty} g\left(g\left(x_{n}\right)\right)=g(x) \text { and } \lim _{n \rightarrow \infty} g\left(g\left(y_{n}\right)\right)=g(y) .
$$

Using the commutativity of $g$ and $\mathrm{G}$ and equation (6), we have

$$
\text { and } \quad \begin{array}{ll}
\mathrm{g}\left(\mathrm{g}\left(\mathrm{x}_{\mathrm{n}+1}\right)\right) & =\mathrm{g}\left(\mathrm{G}\left(\mathrm{x}_{\mathrm{n}}, \mathrm{y}_{\mathrm{n}}\right)\right)=\mathrm{G}\left(\mathrm{g}\left(\mathrm{x}_{\mathrm{n}}\right), \mathrm{g}\left(\mathrm{y}_{\mathrm{n}}\right)\right) \\
\mathrm{g}\left(\mathrm{g}\left(\mathrm{y}_{\mathrm{n}+1}\right)\right) & =\mathrm{g}\left(\mathrm{G}\left(\mathrm{y}_{\mathrm{n}}, \mathrm{x}_{\mathrm{n}}\right)\right)=\mathrm{G}\left(\mathrm{g}\left(\mathrm{y}_{\mathrm{n}}\right), \mathrm{g}\left(\mathrm{x}_{\mathrm{n}}\right)\right) .
\end{array}
$$

Now, we show that $g(x)=G(x, y)$ and $g(y)=G(y, x)$.

Suppose that (i) holds.

Taking limit $\mathrm{n} \rightarrow \infty$ in equations (15) and (16), by equations (13), (14) and continuity of G, we get 


$$
\begin{aligned}
g(x)=\lim _{n \rightarrow \infty} g\left(g\left(x_{n+1}\right)\right)=\lim _{n \rightarrow \infty} G\left(g\left(x_{n}\right), g\left(y_{n}\right)\right) \\
=G\left(\lim _{n \rightarrow \infty} g\left(x_{n}\right), \lim _{n \rightarrow \infty} g\left(y_{n}\right)\right)=G(x, y)
\end{aligned}
$$

and

$$
\begin{aligned}
g(y)=\lim _{n \rightarrow \infty} g\left(g\left(y_{n+1}\right)\right)=\lim _{n \rightarrow \infty} G\left(g\left(y_{n}\right), g\left(x_{n}\right)\right) \\
\quad=G\left(\lim _{n \rightarrow \infty} g\left(y_{n}\right), \lim _{n \rightarrow \infty} g\left(x_{n}\right)\right)=G(y, x),
\end{aligned}
$$

i.e. $g(x)=G(x, y)$ and $g(y)=G(y, x)$.

Suppose that the assumption (ii) holds.

Since $\left\{\mathrm{g}\left(\mathrm{x}_{\mathrm{n}}\right)\right\}$ is non-decreasing and $\mathrm{g}\left(\mathrm{x}_{\mathrm{n}}\right) \rightarrow \mathrm{x}$ and $\left\{\mathrm{g}\left(\mathrm{y}_{\mathrm{n}}\right)\right\}$ is non-increasing and $\mathrm{g}\left(\mathrm{y}_{\mathrm{n}}\right) \rightarrow \mathrm{y}$, we have

$$
\mathrm{g}\left(\mathrm{x}_{\mathrm{n}}\right) \leq \mathrm{x} \text { and } \mathrm{g}\left(\mathrm{y}_{\mathrm{n}}\right) \geq \mathrm{y} \text { for all } \mathrm{n} .
$$

Thus, by FM3, (5), (15) and (16), we have

$\mathrm{M}(\mathrm{g}(\mathrm{x}), \mathrm{G}(\mathrm{x}, \mathrm{y}), \phi(\mathrm{t})) \geq \mathrm{M}\left(\mathrm{g}(\mathrm{x}), \mathrm{g}\left(\mathrm{g}\left(\mathrm{x}_{\mathrm{n}+1}\right)\right), \phi(\mathrm{t})-\phi(\mathrm{qt})\right) * \mathrm{M}\left(\mathrm{g}\left(\mathrm{g}\left(\mathrm{x}_{\mathrm{n}+1}\right)\right), \mathrm{G}(\mathrm{x}, \mathrm{y}), \phi(\mathrm{qt})\right)$

for all $\mathrm{t}>0, \mathrm{q} \in(0,1)$.

Taking $\mathrm{n} \rightarrow \infty$ and solving, we get

$\mathrm{M}(\mathrm{g}(\mathrm{x}), \mathrm{G}(\mathrm{x}, \mathrm{y}), \phi(\mathrm{t})) \geq \mathrm{M}(\mathrm{g}(\mathrm{x}), \mathrm{G}(\mathrm{x}, \mathrm{y}), \mathrm{qt})$.

Taking $\mathrm{q} \rightarrow 1$, which implies that $\mathrm{g}(\mathrm{x})=\mathrm{G}(\mathrm{x}, \mathrm{y})$ by lemma 2.2 \& similarly we can show that $\mathrm{g}(\mathrm{y})=\mathrm{G}(\mathrm{y}, \mathrm{x})$. Thus $\mathrm{G}$ and $\mathrm{g}$ have a coupled coincidence point.

Theorem 3.2. In addition to the hypothesis of theorem 3.1, suppose that for every $(x, y),\left(x^{*}, y^{*}\right) \in X \times X$ there exists a $(\mathrm{u}, \mathrm{v}) \in \mathrm{X} \times \mathrm{X}$ satisfying $\mathrm{g}(\mathrm{u}) \subseteq \mathrm{g}(\mathrm{v})$ or $\mathrm{g}(\mathrm{v}) \subseteq \mathrm{g}(\mathrm{u})$ such that $(\mathrm{G}(\mathrm{u}, \mathrm{v}), \mathrm{G}(\mathrm{v}, \mathrm{u})) \in \mathrm{X} \times \mathrm{X}$ is comparable to $\left(\mathrm{G}\left(\mathrm{x}^{*}, \mathrm{y}^{*}\right)\right.$, $\left.\mathrm{G}\left(\mathrm{y}^{*}, \mathrm{x}^{*}\right)\right)$. Then $\mathrm{G}$ and $\mathrm{g}$ have a unique common coupled fixed point, i.e. there exists a unique $(\mathrm{x}, \mathrm{y}) \in \mathrm{X} \times \mathrm{X}$ such that

$$
x=g(x)=G(x, y), \quad y=g(y)=G(y, x)
$$

Proof. We have to show that if $(x, y),\left(x^{*}, y^{*}\right)$ are couple coincidence points i.e., if $g(x)=G(x, y)$ and $g(y)=G(y, x)$ and $g\left(x^{*}\right)=$ $\mathrm{G}\left(\mathrm{x}^{*}, \mathrm{y}^{*}\right)$ and $\mathrm{g}\left(\mathrm{y}^{*}\right)=\mathrm{G}\left(\mathrm{y}^{*}, \mathrm{x}^{*}\right)$ then

$$
\mathrm{g}(\mathrm{x})=\mathrm{g}\left(\mathrm{x}^{*}\right) \text { and } \mathrm{g}(\mathrm{y})=\mathrm{g}\left(\mathrm{y}^{*}\right) \text {. }
$$

By assumption, there is $(\mathrm{u}, \mathrm{v}) \in \mathrm{X} \times \mathrm{X}$ such that

$(\mathrm{G}(\mathrm{u}, \mathrm{v}), \mathrm{G}(\mathrm{v}, \mathrm{u})) \in \mathrm{X} \times \mathrm{X}$ is comparable to

$G(x, y), G(y, x))$ and $\left(G\left(x^{*}, y^{*}\right), G\left(y^{*}, x^{*}\right)\right)$.

Put $\mathrm{u}_{0}=\mathrm{u}, \mathrm{v}_{0}=\mathrm{v}$ and choose $\mathrm{u}_{1}, \mathrm{v}_{1} \in \mathrm{X}$ so that $\mathrm{g}\left(\mathrm{u}_{1}\right)=\mathrm{G}\left(\mathrm{u}_{0}, \mathrm{v}_{0}\right)$ and $\mathrm{g}\left(\mathrm{v}_{1}\right)=\mathrm{G}\left(\mathrm{v}_{0}, \mathrm{u}_{0}\right)$. We define a sequence $\left\{\mathrm{g}\left(\mathrm{u}_{\mathrm{n}}\right)\right\}$ and $\left\{\mathrm{g}\left(\mathrm{v}_{\mathrm{n}}\right)\right\}$ such that

$$
\mathrm{g}\left(\mathrm{u}_{\mathrm{n}+1}\right)=\mathrm{G}\left(\mathrm{u}_{\mathrm{n}}, \mathrm{v}_{\mathrm{n}}\right) \text { and } \mathrm{g}\left(\mathrm{v}_{\mathrm{n}+1}\right)=\mathrm{G}\left(\mathrm{v}_{\mathrm{n}}, \mathrm{u}_{\mathrm{n}}\right)
$$

These two sequences $\left\{\mathrm{g}\left(\mathrm{u}_{\mathrm{n}}\right)\right\}$ and $\left\{\mathrm{g}\left(\mathrm{v}_{\mathrm{n}}\right)\right\}$ exist and their existence follows from theorem 3.1. In addition, put $\mathrm{x}_{0}=\mathrm{x}, \mathrm{y}_{0}=\mathrm{y}, \mathrm{x}_{0} *=\mathrm{x}$ and $\mathrm{y}_{0} *=\mathrm{y}$ and on the same way, we define the sequence $\left\{\mathrm{g}\left(\mathrm{x}_{\mathrm{n}}\right)\right\}$ and $\left\{\mathrm{g}\left(\mathrm{y}_{\mathrm{n}}\right)\right\}$ and $\left\{\mathrm{g}\left(\mathrm{x}_{\mathrm{n}} *\right)\right\}$ and $\left\{\mathrm{g}\left(\mathrm{y}_{\mathrm{n}} *\right)\right\}$. Since

$\left.(\mathrm{G}(\mathrm{x}, \mathrm{y}), \mathrm{G}(\mathrm{y}, \mathrm{x}))=\left(\mathrm{g}\left(\mathrm{x}_{1}\right), \mathrm{g}\left(\mathrm{y}_{1}\right)\right)\right)=(\mathrm{g}(\mathrm{x}), \mathrm{g}(\mathrm{y}))$ and $(\mathrm{G}(\mathrm{u}, \mathrm{v}), \mathrm{G}(\mathrm{v}, \mathrm{u}))=\left(\mathrm{g}\left(\mathrm{u}_{1}\right), \mathrm{g}\left(\mathrm{v}_{1}\right)\right)$

are comparable, now we assume that $\mathrm{g}(\mathrm{x}) \geq \mathrm{g}\left(\mathrm{u}_{1}\right)$ and $\mathrm{g}(\mathrm{y}) \leq \mathrm{g}\left(\mathrm{v}_{1}\right)$. It is easy to show that $(\mathrm{g}(\mathrm{x}), \mathrm{g}(\mathrm{y}))$ and $\left(\mathrm{g}\left(\mathrm{u}_{\mathrm{n}}\right)\right.$, $\left.\mathrm{g}\left(\mathrm{v}_{\mathrm{n}}\right)\right)$ are comparable, i.e. $\mathrm{g}(\mathrm{x}) \geq \mathrm{g}\left(\mathrm{u}_{\mathrm{n}}\right)$ and $\mathrm{g}(\mathrm{y}) \leq \mathrm{g}\left(\mathrm{v}_{\mathrm{n}}\right)$ for all $\mathrm{n} \geq 1$.

Thus from equation (5), we get

$\mathrm{M}\left(\mathrm{g}(\mathrm{x}), \mathrm{g}\left(\mathrm{u}_{\mathrm{n}+1}\right), \phi(\mathrm{t})\right)=\mathrm{M}\left(\mathrm{G}(\mathrm{x}, \mathrm{y}), \mathrm{G}\left(\mathrm{u}_{\mathrm{n}}, \mathrm{v}_{\mathrm{n}}\right), \phi(\mathrm{t})\right)$

$$
\begin{aligned}
& \geq \mathrm{M}(\mathrm{g}(\mathrm{x}), \mathrm{G}(\mathrm{x}, \mathrm{y}), \mathrm{t}) * \mathrm{M}\left(\mathrm{g}\left(\mathrm{u}_{\mathrm{n}}\right), \mathrm{G}\left(\mathrm{u}_{\mathrm{n}}, \mathrm{v}_{\mathrm{n}}\right), \mathrm{t}\right) \\
& \geq \mathrm{M}\left(\mathrm{g}\left(\mathrm{u}_{\mathrm{n}}\right), \mathrm{g}\left(\mathrm{u}_{\mathrm{n}+1}\right), \mathrm{t}\right) .
\end{aligned}
$$

$\mathrm{M}\left(\mathrm{g}(\mathrm{y}), \mathrm{g}\left(\mathrm{v}_{\mathrm{n}+1}\right), \phi(\mathrm{t})\right)=\mathrm{M}\left(\mathrm{G}(\mathrm{y}, \mathrm{x}), \mathrm{G}\left(\mathrm{v}_{\mathrm{n}}, \mathrm{u}_{\mathrm{n}}\right), \phi(\mathrm{t})\right)$

$$
\begin{aligned}
& \geq \mathrm{M}(\mathrm{g}(\mathrm{y}), \mathrm{G}(\mathrm{y}, \mathrm{x}), \mathrm{t}) * \mathrm{M}\left(\mathrm{g}\left(\mathrm{v}_{\mathrm{n}}\right), \mathrm{G}\left(\mathrm{v}_{\mathrm{n}}, \mathrm{u}_{\mathrm{n}}\right), \mathrm{t}\right) \\
& \geq \mathrm{M}\left(\mathrm{g}\left(\mathrm{v}_{\mathrm{n}}\right), \mathrm{g}\left(\mathrm{v}_{\mathrm{n}+1}\right), \mathrm{t}\right) .
\end{aligned}
$$

for each $n \geq 1$. Letting $n \rightarrow \infty$, we get

$$
\lim _{n \rightarrow \infty} g\left(u_{n+1}\right)=g(x) \text { and } \lim _{n \rightarrow \infty} g\left(v_{n+1}\right)=g(y) .
$$

Similarly, we can prove that 


$$
\lim _{n \rightarrow \infty} \mathrm{g}\left(\mathrm{u}_{\mathrm{n}+1}\right)=\mathrm{g}\left(\mathrm{x}^{*}\right) \text { and } \lim _{\mathrm{n} \rightarrow \infty} \mathrm{g}\left(\mathrm{v}_{\mathrm{n}+1}\right)=\mathrm{g}\left(\mathrm{y}^{*}\right) .
$$

By FM-4, equations (18) and (19), we have

$\mathrm{M}\left(\mathrm{g}(\mathrm{x}), \mathrm{g}\left(\mathrm{x}^{*}\right), \mathrm{t}\right) \geq \mathrm{M}\left(\mathrm{g}(\mathrm{x}), \mathrm{g}\left(\mathrm{u}_{\mathrm{n}+1}\right), \mathrm{t} / 2\right) * \mathrm{M}\left(\mathrm{g}\left(\mathrm{u}_{\mathrm{n}+1}\right), \mathrm{g}\left(\mathrm{x}^{*}\right), \mathrm{t} / 2\right) \rightarrow 1$ as $\mathrm{n} \rightarrow \infty$ which shows that $\mathrm{g}(\mathrm{x})=\mathrm{g}\left(\mathrm{x}^{*}\right)$.

Similarly, we can prove that $\mathrm{g}(\mathrm{y})=\mathrm{g}\left(\mathrm{y}^{*}\right)$.

Since $g(x)=G(x, y)$ and $g(y)=G(y, x)$, by commutativity of $G$ and $g$, we have

$$
\mathrm{g}(\mathrm{g}(\mathrm{x}))=\mathrm{g}(\mathrm{G}(\mathrm{x}, \mathrm{y}))=\mathrm{G}(\mathrm{g}(\mathrm{x}), \mathrm{g}(\mathrm{y}))
$$

and $\quad \mathrm{g}(\mathrm{g}(\mathrm{y}))=\mathrm{g}(\mathrm{G}(\mathrm{y}, \mathrm{x}))=\mathrm{G}(\mathrm{g}(\mathrm{y}), \mathrm{g}(\mathrm{x}))$.

Say $\mathrm{g}(\mathrm{x})=\mathrm{z}, \mathrm{g}(\mathrm{y})=\mathrm{w}$, therefore, from equations (20) and (21)

$$
\mathrm{g}(\mathrm{z})=\mathrm{G}(\mathrm{z}, \mathrm{w}) \text { and } \mathrm{g}(\mathrm{w})=\mathrm{G}(\mathrm{w}, \mathrm{z}) .
$$

Thus, $(\mathrm{z}, \mathrm{w})$ is a coupled common fixed point. Then from equation (17) with $\mathrm{x}^{*}=\mathrm{z}$ and $\mathrm{y}^{*}=\mathrm{w}$, it follows that $\mathrm{g}(\mathrm{z})=\mathrm{g}(\mathrm{x})$ and $\mathrm{g}(\mathrm{w})=$ $\mathrm{g}(\mathrm{y})$, i.e.

$$
\mathrm{g}(\mathrm{z})=\mathrm{z} \text { and } \mathrm{g}(\mathrm{w})=\mathrm{w} .
$$

Now from equations (22) and (23),

$$
\mathrm{z}=\mathrm{g}(\mathrm{z})=\mathrm{G}(\mathrm{z}, \mathrm{w}) \text { and } \mathrm{w}=\mathrm{g}(\mathrm{w})=\mathrm{G}(\mathrm{w}, \mathrm{z}) .
$$

Therefore, $(\mathrm{z}, \mathrm{w})$ is a coupled common fixed point of $\mathrm{G}$ and $\mathrm{g}$.

To prove the uniqueness, assume that (p, q) is another coupled common fixed point. Then from equation (17), we have

$$
\mathrm{p}=\mathrm{g}(\mathrm{p})=\mathrm{g}(\mathrm{z})=\mathrm{z} \text { and } \mathrm{q}=\mathrm{g}(\mathrm{q})=\mathrm{g}(\mathrm{w})=\mathrm{w} .
$$

This completes the proof.

If we take $\mathrm{g}=\mathrm{I}$, then we get Corollary 3.1 :

Corollary 3.1. Let $(X, M, *)$ be a complete fuzzy metric space and $(X, \leq)$ be a partially ordered set and let $G: X \times X \rightarrow X$ such that $\mathrm{G}$ has a mixed monotone property and there exists $\phi \in \Phi$ such that

$\mathrm{M}(\mathrm{G}(\mathrm{x}, \mathrm{y}), \mathrm{G}(\mathrm{u}, \mathrm{v}), \phi(\mathrm{t})) \geq \mathrm{M}(\mathrm{x}, \mathrm{G}(\mathrm{x}, \mathrm{y}), \mathrm{t}) * \mathrm{M}(\mathrm{u}, \mathrm{G}(\mathrm{u}, \mathrm{v}), \mathrm{t})$

for all $\mathrm{x}, \mathrm{y}, \mathrm{u}, \mathrm{v} \in \mathrm{X}, \mathrm{t}>0$ for which

or

$$
\mathrm{x} \geq \mathrm{u} \text { and } \mathrm{y} \leq \mathrm{v}
$$

Suppose either

(iii)

$\mathrm{G}$ is continuous or

(iv) $\mathrm{X}$ has the following properties :

(c) If a non-decreasing sequence $\mathrm{x}_{\mathrm{n}} \rightarrow \mathrm{x}$, then $\mathrm{x}_{\mathrm{n}} \leq \mathrm{x}$, for all $\mathrm{n}$ and

(d) If a non-increasing sequence $y_{n} \rightarrow y$, then $y_{n} \geq y$, for all $n$.

If there exist $\mathrm{x}_{0}, \mathrm{y}_{0} \in \mathrm{X}$ such that

$$
\mathrm{x}_{0} \leq \mathrm{G}\left(\mathrm{x}_{0}, \mathrm{y}_{0}\right), \quad \mathrm{y}_{0} \geq \mathrm{G}\left(\mathrm{y}_{0}, \mathrm{x}_{0}\right)
$$

then there exist $\mathrm{x}, \mathrm{y} \in \mathrm{X}$ such that

$$
\mathrm{x}=\mathrm{G}(\mathrm{x}, \mathrm{y}) \text { and } \mathrm{y}=\mathrm{G}(\mathrm{y}, \mathrm{x}) .
$$

Then $\mathrm{G}$ has a coupled coincidence point in $\mathrm{X}$.

Now taking $\phi(\mathrm{t})=\mathrm{kt}$ in theorem 3.1 , we get Corollary 3.2 .

Corollary 3.2. Let $(X, M, *)$ be a complete fuzzy metric space and $(X, \leq)$ be a partially ordered set and let $G: X \times X \rightarrow X$ and $\mathrm{g}: \mathrm{X} \rightarrow \mathrm{X}$ be two mappings such that $\mathrm{G}$ has a mixed $\mathrm{g}$-monotone property and there exists $\mathrm{k} \in(0,1)$ such that

$$
\mathrm{M}(\mathrm{G}(\mathrm{x}, \mathrm{y}), \mathrm{G}(\mathrm{u}, \mathrm{v}), \mathrm{kt}) \geq \mathrm{M}(\mathrm{g}(\mathrm{x}), \mathrm{G}(\mathrm{x}, \mathrm{y}), \mathrm{t}) * \mathrm{M}(\mathrm{g}(\mathrm{u}), \mathrm{G}(\mathrm{u}, \mathrm{v}), \mathrm{t})
$$

for all $\mathrm{x}, \mathrm{y}, \mathrm{u}, \mathrm{v} \in \mathrm{X}, \mathrm{t}>0$ for which

$$
\mathrm{g}(\mathrm{x}) \geq \mathrm{g}(\mathrm{u}) \text { and } \mathrm{g}(\mathrm{y}) \leq \mathrm{g}(\mathrm{v})
$$

or

$$
\mathrm{g}(\mathrm{x}) \leq \mathrm{g}(\mathrm{u}) \text { and } \mathrm{g}(\mathrm{y}) \geq \mathrm{g}(\mathrm{v}) \text {. }
$$

Suppose $\mathrm{G}(\mathrm{X} \times \mathrm{X}) \subseteq \mathrm{g}(\mathrm{X})$ is continuous and commutes with $\mathrm{G}$ and also suppose either 
(v) $\mathrm{G}$ is continuous or

(vi) $\mathrm{X}$ has the following properties :

(e) If a non-decreasing sequence $x_{n} \rightarrow x$, then $x_{n} \leq x$, for all $n$ and

(f) If a non-increasing sequence $y_{n} \rightarrow y$, then $y_{n} \geq y$, for all $n$.

If there exist $\mathrm{x}_{0}, \mathrm{y}_{0} \in \mathrm{X}$ such that

$$
\mathrm{g}\left(\mathrm{x}_{0}\right) \leq \mathrm{G}\left(\mathrm{x}_{0}, \mathrm{y}_{0}\right), \mathrm{g}\left(\mathrm{y}_{0}\right) \geq \mathrm{G}\left(\mathrm{y}_{0}, \mathrm{x}_{0}\right)
$$

then there exist $\mathrm{x}, \mathrm{y} \in \mathrm{X}$ such that

$$
\mathrm{g}(\mathrm{x})=\mathrm{G}(\mathrm{x}, \mathrm{y}) \text { and } \mathrm{g}(\mathrm{y})=\mathrm{G}(\mathrm{y}, \mathrm{x}) .
$$

Then $\mathrm{G}$ and $\mathrm{g}$ have a coupled coincidence point in $\mathrm{X}$.

Corollary 3.3. Assume hypothesis of Corollary 3.1 holds. If $x_{0}$ and $y_{0}$ are comparable, then $x=y$, where $x$ and $y$ are the coupled fixed point of $\mathrm{G}$, i.e, $\mathrm{x}=\mathrm{G}(\mathrm{x}, \mathrm{x})$.

\section{REFERENCES}

[1] Bhaskar, T.G. and Lakshmikantham, V., Fixed point theorem in partially ordered metric spaces and applications, Nonlinear Anal. 65 (2006), 1379-1393.

[2] Deng, Z. K., Fuzzy pseduo-metric spaces, J.Math. Anal. Appl. 86(1982), 191-207.

[3] Erceg, M. A., Metric spaces in fuzzy set theory, J.Math. Anal. Appl. 69(1979), 205-230.

[4] George, A. and Veeramani, P., On some results in fuzzy metric spaces, Fuzzy Sets and System 64(1997), 395399.

[5] Kaleva, O. and Seikkala, S., On fuzzy metric spaces, Fuzzy Sets and Systems 12(1984), 215-229.

[6] Kramosil, I. and Michalek, J., Fuzzy metric statistical metric spaces, Kybernetica 11(1975), 326-334.

[7] Lakshmikantham, V. and Ćirić, Lj.B., Coupled fixed point theorem for nonlinear contractions in partially ordered metric spaces, Nonlinear Anal. 70 (2009), 4341-4349.

[8] Zadeh, L. A., Fuzzy Sets, Inform. and Control 89(1965), 338-353.

\section{Biography -}
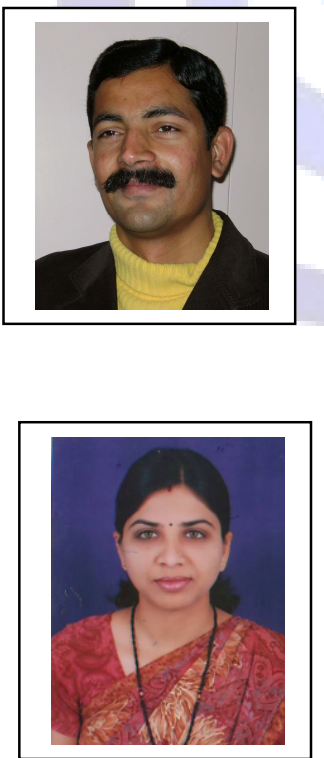

Dr. Arihant Jain did his Post Graduation in Mathematics in the year 2000 from School of Studies in Mathematics, Vikram University, Ujjain. He has been awarded doctorate degree in Mathematics in the year 2007 from School of Studies in Mathematics, Vikram University, Ujjain on Fixed Point Theory. He has published 100 research papers in national and international journals of repute. He has a post graduate teaching experience of 7 years and graduate teaching experience of 6 years. Two students have got Ph.D. under his guidance. Presently, he is working on the post of Academic Dean, Professor and Head, Department of Applied Mathematics, Shri Guru Sandipani Institute of Technologv and Science. I Jiiain and activelv engaged with his researchers.

Ms. Vaijayanti Supekar did M.Sc. in Mathematics from Holkar Science College, Indore in the year 2005 and B.Ed. from IGNOU in 2011. Presently, he is working on the post of Assistant Professor (Mathematics) at Malwa Institute of Technology Indore (M.P.) and currently pursuing Ph.D. from Mewar University, Chittorgarh. 